\title{
A Highly-Reliable Full-Automatic System for Analyzing ECG Waveforms in Real Time Applications
}

\author{
Antoun Khawaja ${ }^{1}$ \\ ${ }^{1}$ Khawaja Medical Technology GmbH, Gräfelfing near Munich, Germany
}

\begin{abstract}
The ECG analysis system, presented in this paper, provides beat-to-beat localization, classification and measurements in real time. Besides, numbers of rhythm analysis events can be detected instantly by the system, including critical ventricular and atrial arrhythmia events. Using the system will increase the cardiac safety for patients in many cardiac applications, including home-monitoring, ambulatory monitoring and cardiac drug safety. The algorithms used by the system are validated and tested. Furthermore, the system can be deployed on different computing hardware targets and operation systems.
\end{abstract}

\section{Introduction}

ECG diagnosis is essential for recognition and treatment of cardiac diseases. Diagnosis of the electrocardiogram can be realized either manually, semi-automatically or full-automatically. Furthermore, it can be carried out offline after gathering ECG signal or live during the recording. Real-time detection of serious cardiac trends and life-threatening cardiac events allows providing immediate medical care and therapy for patients. As a result, the cardiac safety in many cardiac applications will be improved including home-monitoring, ambulatory monitoring and cardiac drug safety. In this work, a full-automatic and high reliable ECG analysis system in real-time is presented. It can provide relevant and important ECG biomarkers, measurements and events. The algorithms employed in this system perform reliable quality-assessment of ECG signals, robust detection of the QRS complexes based on time-frequency analysis [1], highly precise classification of QRS complex based on machine-learning methodologies [1], highly accurate full-scale beat-to-beat delineation of heart beats [2], as well as numerous statements and measures for ECG rhythm analysis, respectively. The system can handle single-lead ECG as well as multi-channel ECG lead systems.

\section{Methods}

\subsection{System requirements}

The ECG analysis system presented in this paper has numbers of essential requirements, which are listed as follows:

- Platform independent: The system along with its components and units is designed in a generic architecture to be deployed on different hardware targets and operation systems including Windows, Mac OS X, Linux, Android and iOS. The main objective here is to develop once and deploy everywhere.

- Channel independent: The system along with its components and units is designed to handle single-channel as well as multi-channel lead schemes. Number of available input ECG leads is recognized automatically by the system.

- Sample-frequency independent: The system along with its components and units is designed to handle different input ECG signals digitalized in different sample frequency. However, the minimal sample frequency the system can deal with is $125 \mathrm{~Hz}$.

- Configurable and maintainable: The system along with its components and units can be configured by numbers of input config values making the system easy to adjust for users' needs. Furthermore, it allows easy maintenance of system components.

- Beat-to-Beat analysis: The system along with its components and units is engineered to carry out analysis based on every single heart beat. This will allow a full scope and high resolution analysis for the whole ECG data.

- Regulatory compliance: The system along with its components and units is developed, validated and verified according to the harmonized international standards for medical devices and medical software including ISO 13485, IEC 62304 and ISO 13971.

- Model-based design: The system along with its components and units is developed, validated and verified according to the design methodology, which is based on developing so-called models and sub-models. The model-based design is significantly different from traditional design methodology. Rather than using complex structures and 
extensive software code, developers can use model-based design to define models with advanced functional characteristics using so-called building blocks and elements. These built models used with simulation tools can lead to rapid prototyping, software testing, and verification. Not only is the testing and verification process enhanced, but also, in some cases, hardware-in-the-loop simulation can be used with the new design paradigm to perform testing of dynamic effects on the system more quickly and much more efficiently than with traditional design methodology. Figure 1 shows the top model as well as the related submodels and components of the ECG analysis system presented in this work.

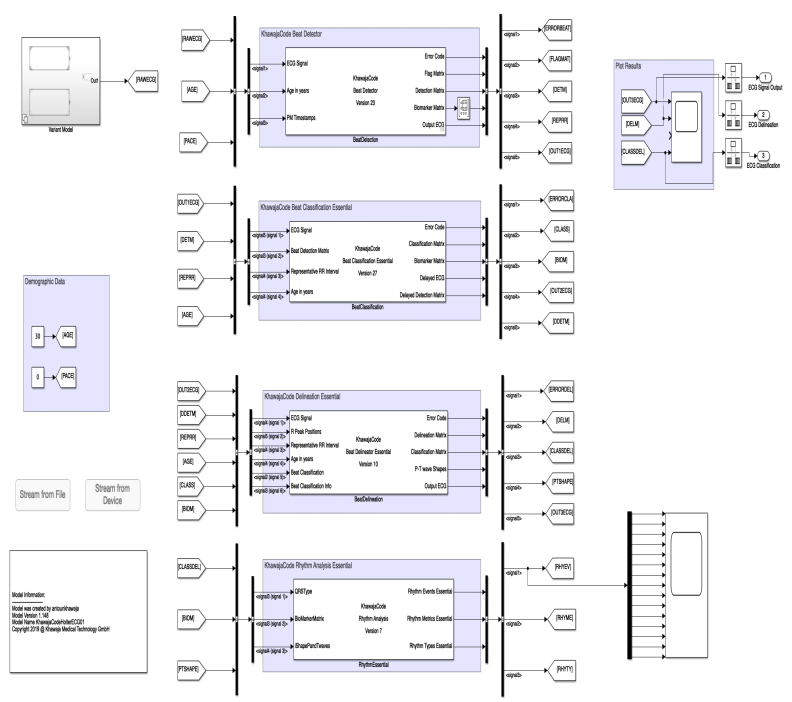

Figure 1. The top model as well as the related sub-models and components of the ECG analysis system presented in this work.

- Modular design: The architecture of the system along with its components and units is designed according to an approach that subdivides the whole system into smaller parts called modules. This design can be characterized by functional partitioning into discrete scalable, reusable modules as well as use of well-defined modular interfaces. - ECG analoge front-end compatibility: ECG analog front-end is a hardware component, which is responsible to capture ECG signals from human body by means of ECG electrodes, amplify those signals and finally digitalize them using appropriate Analog-to-Digital Converter (ADC). This hardware component is not a part of the software system presented here. The digital output (digital ECG data) of such component is the input of our system. Any ECG analog front-end can be utilized with this ECG analysis system. However, for validation and verification purposes, the12-channel ECG analog front-end EG12000 provided by the company MedLab [3] is used in this work.

\subsection{Development environment}

MATLAB language and SIMULINK Integrated Development Environment (IDE) along with the relevant toolboxes provided by the company The MathWorks, Inc. [4] are used for the whole development, implementation and test for the ECG analysis system described here. Development activities and actions are carried out according to the so-called reference workflow for the model-based development of safety and critical software [6]. This workflows is provided by The MathWorks, Inc. and precertified for medical software development by TÜV SÜD, a German certification authority [5]. This reference workflow provides mapping to IEC 62304 and describes modelbased development including automatic code generation and model- based testing methods.

- Design and Simulation: The whole model including components and algorithms that affects system behavior is design, developed and validated in native low-level MATLAB language supported for $\mathrm{C} / \mathrm{C}++$ code generation and domain-specific modeling tools. Simulation is carried out using SIMULINK environment and specific toolboxes. This will avoid expensive, risky, or time-consuming analysis of the system performance.

- Implementation: Using code generation tools in MATLAB and SIMULINK, C, C++ or target-specific executable libraries can be automatically generated from the whole model including components and algorithms. The generated code can be utilized for rapid prototyping or production.

- Test and Verification: Using test tools in MATLAB and SIMULINK, reusable test harness for virtual integration and hardware-in-the-loop testing for the ECG analysis system are provided.

\subsection{System components and architecture}

\subsubsection{Preprocessing component}

This software component carries out many essential steps:

- Collect ECG data from the analog front-end in real time

- Precondition ECG data and check the plausibility of the data

- Perform quality check on ECG data

\subsubsection{Heart beat detection}

This software component employs the algorithm responsible for heart beat detection [1]. The methodology of identifying ECG heart beats is developed based on timefrequency analysis. Besides, it carries out numbers of plausibility checks to ensure the accurate detection of the physiological heart cycles. This algorithm is validated using 
MIT-BIH Arrhythmia Database, AHA Series1 and AHA Series 2 and obtained sensitivity and specificity values greater than $99.7 \%$ in both measures and in all databases. The software component provides, in addition to heart beat locations, coarse position of QRS onset and QRS offset for each cycle. Figure 2 shows an example for heart beat detection done on a randomly-chosen real-world ECG signal in real time using this software component. Figure 3 shows Poincaré plot for each RR interval on $\mathrm{x}$-axis versus succeeding RR interval on y-axis detected on a real-world long-term ECG signal by this software component in real time.

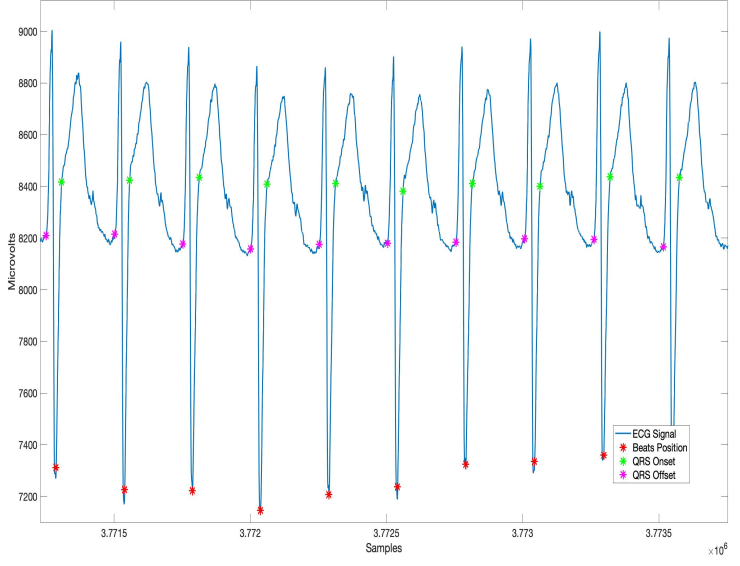

Figure 2. An example for heart beat detection done on a randomly-chosen real-world ECG signal in real time using the software component for heart beat detection.

\subsubsection{Heart beat classification}

This software component employs the algorithm responsible for heart beat classification [1]. The methodology of classifying ECG heart beats is developed based on machine-learning techniques. The algorithm can classify detected heart beats into five main classes, namely Normal for normal beats, $P V C$ for premature ventricular contraction beats, $P S C$ for premature supra-ventricular contraction beats, Paced for paced beats and Unclassified for beats that cannot be classified. This algorithm is validated using MIT-BIH Arrhythmia Database, AHA Series1 and AHA Series 2 and obtained sensitivity and specificity values greater than $93.5 \%$ and $96.5 \%$ in all databases, respectively. Figure 4 shows a waterfall plot for all detected heart beat classified as Normal in a a real-world long-term ECG signal using the heart beat classification algorithm in real time.

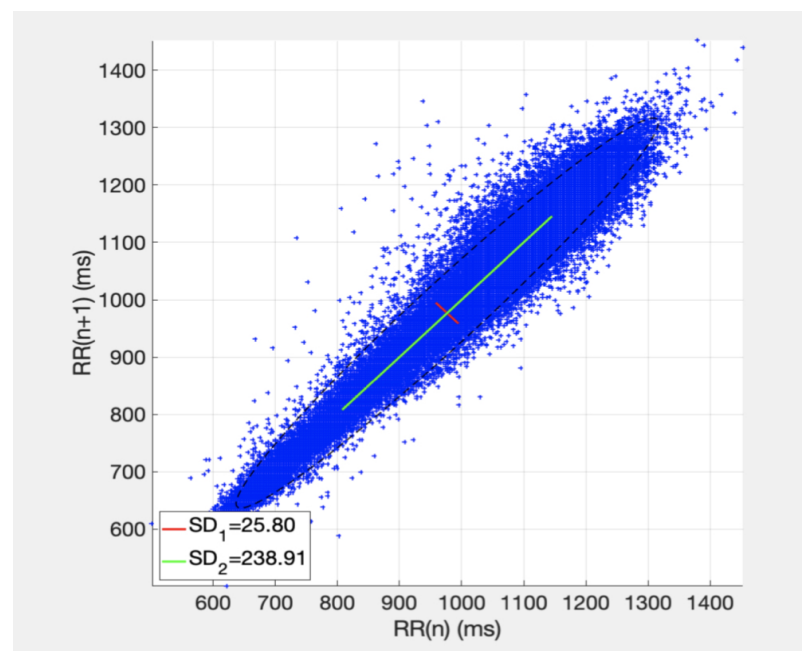

Figure 3. Poincaré plot for each RR interval on $x$-axis versus succeeding RR Interval on y-axis detected on a realworld long-term ECG signal using the software component for Heart beat detection in real time.

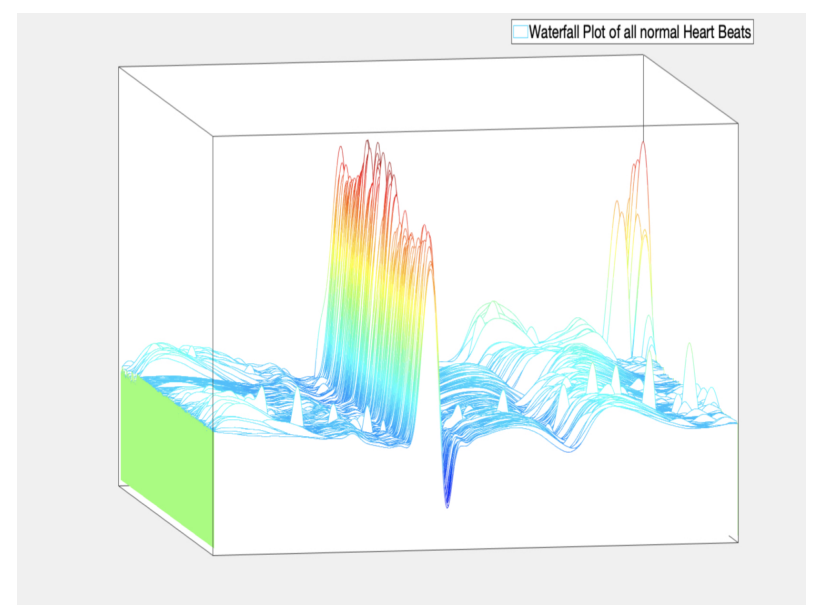

Figure 4. A waterfall plot for all detected heart beat classified as Normal in a a real-world long-term ECG signal using the heart beat classification algorithm in real time.

\subsubsection{Heart beat delineation}

This software component employs the algorithm responsible for detecting the essential fiducial points and measuring the segments and intervals of each detected heart beat [1]. The fiducial points localized here are $P$ wave onset, P wave offset, QRS complex onset, QRS complex offset and $\mathrm{T}$ wave offset. Whereas, the segments and intervals are PQ / PR segment, ST segment, PR interval, QRS inter$\mathrm{val} /$ duration and QT interval. This algorithm is validated and tested according to the procedure and databases described in the standard IEC 60601-2-25:2011. All relevant requirements of the standard have been fulfilled by the de- 
lineation algorithm. The delineation results illustrates a high degree of agreement with the reference annotations done in CTS and CSE and the delineation results provided by the Heart Beat Delineation Algorithm [2]. Figure 5 shows Poincaré scatter plot for each RR interval on $\mathrm{X}$-axis versus QT interval on y-axis detected on a real-world longterm ECG signal by this software component in real time.

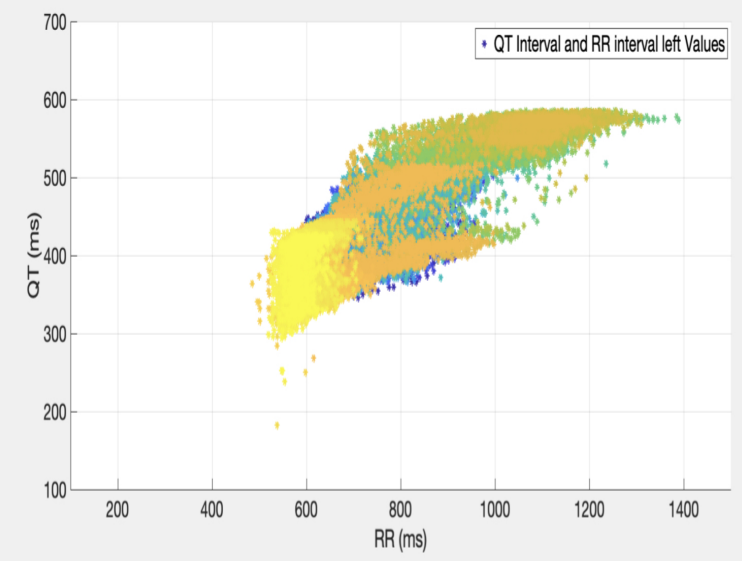

Figure 5. Poincaré scatter plot for each RR interval on $\mathrm{X}-$ axis versus QT interval on y-axis detected on a real-world long-term ECG signal by the heart beat delineation component in real time.

\subsubsection{Heart rhythm analysis}

This software component employs the algorithm responsible for providing rhythm analysis based on beat detection, beat classification, beat delineation and ECG morphology. The algorithm can provide numbers of rhythm events including Bigeminy, Trigemini, Couplet, Triplet, Salve / Run, Ventricular Tachycardia, Sinus Tachycardia, Sinus Bradycardia, Asystoly and Cardiac Pause, Ventricular Fibrillation, Atrial Fibrillation, A-V Blocks, WPW Syndrom, Respiration Event in ECG. Figure 6 shows an example of Trigemini events found by the algorithm of the heart rhythm analysis in a long-term ECG signal, where $\mathrm{x}$-axis represents ECG recording time in 5-minute periods and y-axis represent Trigemini event flags. The Ventricular Fibrillation and Atrial Fibrillation are still under validation process, whereas the rest are already validated.

\section{Discussion and conclusion}

Due to its high degree of performance and accuracy, the system can be used in critical cardiac applications including cardiac drug safety minimizing the hazard and risk to

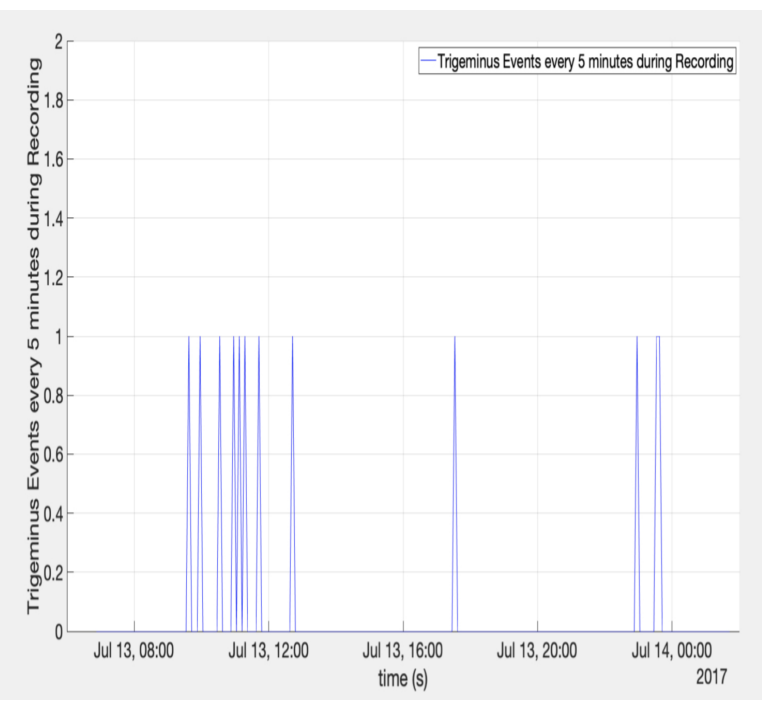

Figure 6. Trigemini events found by the algorithm of the heart rhythm analysis in a long-term ECG signal, where $\mathrm{x}$-axis represents ECG recording time in 5-minute periods and $y$-axis represent Trigemini event flags.

patients and increasing the cardiac safety and security in total. Using 12-channel ECG lead system, additional diagnosis component can be added to this system. This component can provide extended atrial and ventricular diagnosis as well as repolarization and cardiac conduction system diagnosis. Moreover, statements about ischemia and myocardial infarction can be provided in real-time by using the validated diagnostic algorithm, presented in [1], as an add-on to this system.

\section{References}

[1] A. Khawaja, "A Novel Algorithm for Full-Automatic ECG Interpretation and Diagnostics", in Proc. Computers in Cardiology, vol. 45, ISSN: 2325-887X DOI: 10.22489/CinC.2018.229, 2018.

[2] A. Khawaja, "A Novel Algorithm for Full-Automatic Multipurpose ECG Delineation," in Proc. Computers in Cardiology, vol. 45,ISSN: 2325-887X DOI: 10.22489/CinC.2018.209, 2018.

[3] MEDLAB medizinische Diagnosegeräte $\mathrm{GmbH}$, https://www.medlab.eu

[4] The MathWorks, Inc., https://www.mathworks.com

[5] TÜV SÜD GmbH, https://www.tuev-sued.de/

[6] Reference Workflow, https://de.mathworks.com/help/certkitiec/ ug/embedded-coder-reference-workflow-overview.html

Address for correspondence:

Dr. Antoun Khawaja

Am Haag 8, 82166 Gräfelfing near Munich, Germany

Antoun.Khawaja@khawaja-medtech.com 